\title{
B-cell Lymphoma as a Complication of Sjogren's Syndrome: A Case Report
}

ISSN: 2639-0531

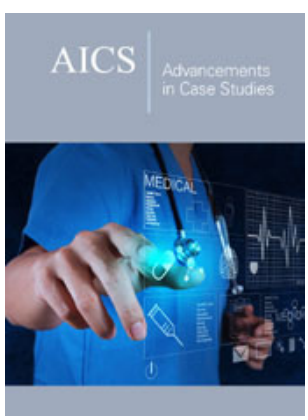

*Corresponding author: Piazera FZ, Medical Department, Brazil

Submission: 眥 January 21, 2020

Published: 侮 February 11, 2020

Volume 2 - Issue 3

How to cite this article: Costa SM, Perdigao AG, Luz WC, Piazera FZ. B-cell Lymphoma as a Complication of Sjogren's Syndrome: A Case Report. Adv Case Stud.2(3). AICS.000539.2020. DOI: 10.31031/AICS.2020.02.000539

Copyright@Piazera FZ, This article is distributed under the terms of the Creative Commons Attribution 4.0 International License, which permits unrestricted use and redistribution provided that the original author and source are credited.

\author{
Costa SM, Perdigao AG, Luz WC and Piazera FZ*
}

Medical Department, Brazil

\begin{abstract}
Sjogren's Syndrome is an autoimmune dysfunction of the exocrine glands that causes dryness of the eyes, mouth, skin. One of its worst complications is MALT lymphoma, which is caused by deregulation of B-cell proliferation. In this case report a female patient with primary Sjogren's Syndrome presents a parotid swelling, concluded by biopsy that was a lymphoma. After resection, she was treated with rituximab. The immunotherapy caused the remission of the lymphoma and the symptoms of the primary disease. The use of immunotherapy might be an alternative to the traditional immunosuppressants used for Sjogren's Syndromea.
\end{abstract}

Keywords: Sjogren's syndrome; Sicca syndrome; B-cell lymphoma; Rituximab; Case report

Abbreviations: SS: Sjogren's Syndrome; PET Scan: Positron Emission Tomography

\section{Introduction}

Sjogren's Syndrome (SS) is a dysfunction of the exocrine glands in response to lymphocytic infiltration and deposition of immune complexes, which leads to a chronic condition and a progressive glandular destruction. Sicca Syndrome, as it is called, impairs the production of teardrops, saliva, sweat and mucus, causing the dryness present in the disease. SS has a low prevalence in the general population and is more prevalent in women (9:1), mainly in the fourth and fifth decade of life.

Despite the benign chronic outcome, one of the complications of the disease is the development of lymphoma. It is up to 40 times more likely to develop lymphoma in a patient with Sjogren's Syndrome than in the general population [1]. Lymphocytic infiltration and autoantibody production create a likely environment to turn polyclonal SS lymphoproliferation into monoclonal proliferation, resulting in malignancy. The inflammatory state enhances apoptosis mechanisms and hyper-stimulated B lymphocytic proliferation. The objective of this paper is to report a case of a patient who presented parotid lymphoma secondary to Sjogren's Syndrome and was treated with rituxumab after a partial parotidectomy.

\section{Case Report}

Female patient, 49 years old, diagnosed with primary Sjogren's Syndrome (being the diagnostic clinical criteria xerostomia, dry eye, parotid hypertrophy and in the biopsy of parotid gland) in 2017, treated with azathioprine and prednisone for control [2]. After 2 years of diagnose, she presented rapid growth of the right parotid. In May 2019, she underwent a partial right parotidectomy for biopsy. An immunohistochemical examination and the clinical signs indicated a B-cell lymphoma. (Figure 1). Serological tests for hepatitis C and B as well as HIV were negative. The patient had no previous use of antibiotics or a history of infection with the mumps virus.

The anatomopathological report showed extra-nodal marginal zone lymphoma (MALT lymphoma) of the salivary gland, originating in the context of lymphoepithelial sialodenitis (" LESA "). Larger salivary gland parenchyma and architecture was extensively damaged by the presence of dense lymphoid proliferation, predominantly of small cells, in which multiple foci of lymphoepithelial lesions (epimioepithelial islands) are observed [3]. B lymphoid cells showed frequent monocytoid appearance and formed collars and anastomosing sheets around the lymphoepithelial lesions. A marked atrophy of the acinar epithelium with lymphoid proliferation was also observed permeating nerve threads and extending to the peri-glandular adipose tissue. 
In July 2019, the patient was evaluated by the oncohematology service, where she underwent clinical training with PET-CT. The PET-CT showed a moderate diffuse increase in parotid metabolism, related to the Sjogren Syndrome inflammatory activity and the surgical manipulation. No more lymphoproliferative tissue was found after the resection. (Figure 2 \& 3). After all evaluations, with the physical examination findings, laboratory data and PETCT result, the patient presented with IE staging according to the Ann Arbor classification, modified by Lugano. in view of the risk of worsening symptoms of sicca syndrome, we opted to undergo treatment with monotherapy, due to literature data on a high index of local relapse or second contralateral lymphoma in the left parotid [4]. The adjuvant treatment was four cycles of Mab Thera (rituximab) started in August 2019. The patient evolved with complete lymphoma response and, luckly, markedly reduced the symptoms of Sjogren's Syndrome, keeping disease in remission without the use of immunosuppressants.

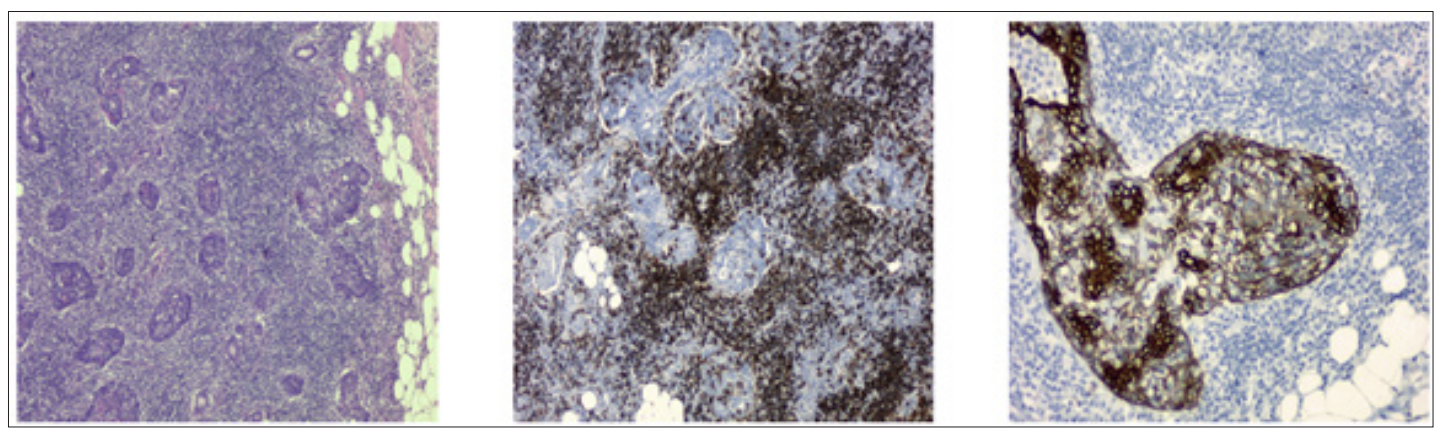

Figure 1: Immunohistochemical exam shows damaged salivary gland tissue, lymphoid proliferation.

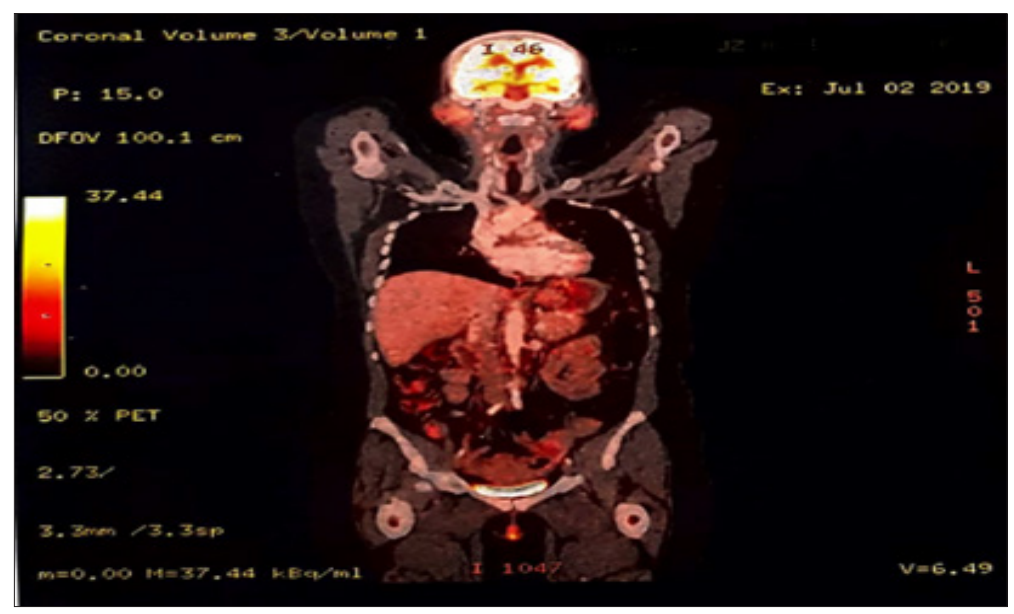

Figure 2:

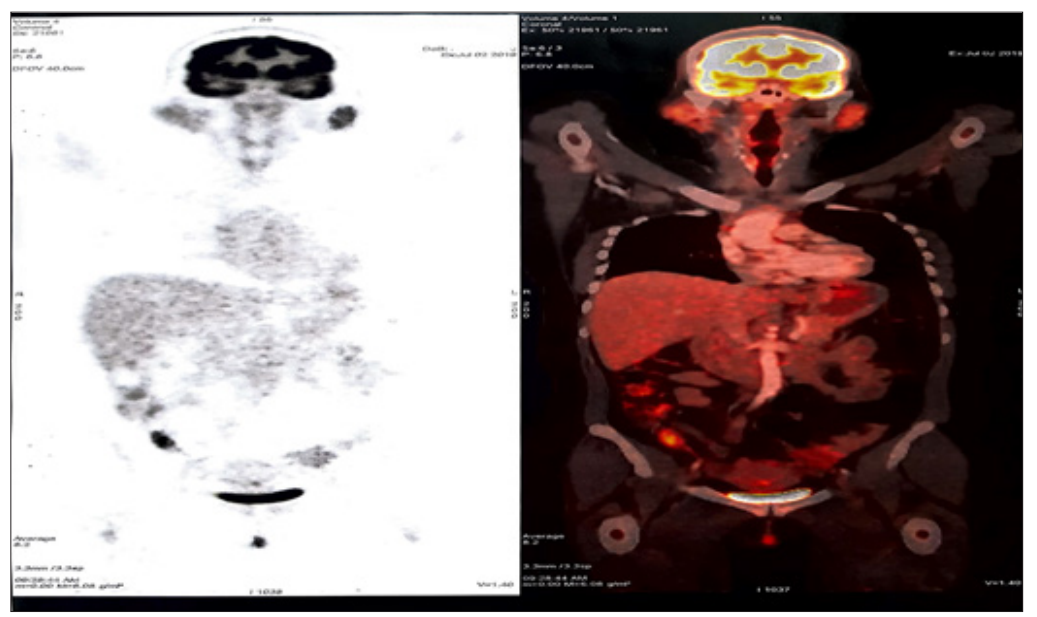

Figure 3: 
Figure 1 and 2 PET-CT: Examination performed with the intravenous administration of $12.92 \mathrm{mCi}$ fluorine-2-desoxyglucose marked fluorine 18 (18F). Blood glucose $69 \mathrm{mg} / \mathrm{dl}$ before injection. Images taken 60 minutes after the drug injection using a 16-channel PET-CT, free breathing, iodine marker. Head and neck images suggest surgical manipulation of the right parotid gland, without hypermetabolism areas, but diffuse hypermetabolism in both glands caused by Sjogren's Syndrome. Hypertrophy of nasopharynx lymphoid tissue. Lymph nodes in normal size, shape and activity.

\section{Discussion}

Sjogren's Syndrome is an autoimmune systemic disease characterized by lymphocyte infiltration into secretory glands causing dryness of the eyes, mouth, larynx, pharynx and vagina. Patients may also develop systemic manifestations in renal, pulmonary and neurological systems. Despite presenting a favorable evolution, it may be a predisposing factor for the development of potentially dangerous lymphoproliferative processes. Constant vigilance for signs or symptoms of a malignant processes - such as rheumatoid factor dosing, persistence of antibodies, or onset of systemic symptoms unresponsive to treatment - is needed to early diagnose.

These patients are twice more at risk of developing lymphoma than the general population, because lymphocytic infiltration and the production of auto-AC generate persistent glandular involvement facilitating the transformation into low-grade B-cell lymphomas. Development usually occurs between 4 and 12 years of the disease and up to $5 \%$ of patients may present lymphoma. Lymphoma development is facilitated by higher levels of B-cell activation factor, which is related to increased disease activity in these glands. Polyclonal lymphoproliferation that characterizes the syndrome may evolve into monoclonal and then in malignancy. Factors implicated in the genesis of lymphoma include dysregulation of the mechanisms that lead to apoptosis, hyperstimulation of $\mathrm{B}$ cells and participation of infectious agents, such as hepatitis $C$ virus. Another risk factor is the use of steroids and immunosuppressive drugs, which constitute the basis of the treatment of Sjogren's Syndrome. So, the treatment itself bias the onset of lymphoma.

The therapy used to treat the patient with the syndrome and the lymphoma is preferably made with drugs that modify the course of the disease, such as methotrexate, but the use of immunebiologicals show the most satisfactory results, among them the most used is Rituximab, an anti-CD20 drug that blocks the action of B lymphocytes. In the case report, we have a patient diagnosed with Sjogren's Syndrome two years ago who having presented a prominent swelling in the topography region of the parotid gland, underwent investigation and partial removal of the gland. In post-excisional biopsy, B-cell lymphoma was confirmed. After resection and pharmacological treatment, the immunosuppressant played a role in the remission of lymphoma and of symptoms of the primary disease - enabling a period of time free from the symptoms of the disease without the regular medications. The use of immunotherapy might be a way out of the traditional treatments for autoimmune diseases such as Sjogren's Syndrome, making possible an intermittent treatment with less side effects and lower risk of complications such as MALT lymphoma.

\section{References}

1. Falero YB, Cartas US, Hernández AA, Hernández YA (2016) Association between Sjögren's syndrome and tumores: about a case. Rev Archivo Médico De Camaguey 20(2).

2. Fragkioudaki S, Mavragani C, Moutsopoulos H (2016) Predicting the risk for lymphoma development in Sjogren syndrome. Medicine 95(25): e3766.

3. Limares FDC, Soler RC, Filho IB (2005) Lymphoma manifestations in Sjogren's syndrome: is there a relationship? Rev Bras Otorrinolaringol $71(3)$.

4. Nocturne G, Pontarini E, Bombardieri M, Mariette X (2019) Lymphomas complicating primary Sjogren's syndrome: from autoimmunity to lymphoma. Reumathology.

For possible submissions Click below: 